\title{
Social-emotional development in early life: what happens and how to optimize it
}

Received 18 November 2021 Accepted 4 January 2022

Link to DOI:

10.25220/WNJ.V05.S2.0002

Journal Website: www.worldnutrijournal.org

\author{
Bernie Endyarni Medise ${ }^{1}$, Tjhin Wiguna ${ }^{2}$, Ni Ketut Susila Dharma Asih ${ }^{3}$ \\ 1. Child Health Department, Faculty of Medicine, Universitas Indonesia \\ 2. Department of Psychiatry, Faculty of Medicine, Universitas Indonesia \\ 3. Danone Specialized Nutrition Indonesia
}

\begin{abstract}
The Physical, metabolic, emotional, cognitive, and social development all begin to develop during early years of childhood.. Social and emotional competencies are increasingly recognized as critical for children's success, in school and in later phases of life into adulthood. According to new research, changes in the gastrointestinal tract's development during the early postnatal period can affect brain development and vice versa., collectively called the gut-brain axis. The gut microbiota has an impact on a variety of mental processes and phenomena, as well as being involved in the pathophysiology of a variety of mental and neurological diseases.. Insights in this area can be targeted through dietary treatments to improve cognitive outcomes in newborns by optimizing the link between the gastrointestinal system and the brain. Further, having a healthy and happy human life could be ensured by acquiring adequate and balance microbiota.

Keywords gut brain axis, gut microbiota, socio-emotional
\end{abstract}

\section{Introduction}

Early childhood is characterized by the successful acquisition of social-emotional skills. ${ }^{1,2}$ Early childhood research has highlighted the importance of a child's first five years of life on his/her social emotional development. ${ }^{3}$ The basis for perceptual, cognitive, and emotional capacities is laid during the early postnatal period, which is known as critical for the creation of long-term cognitive and behavioral talents. . Recently, the first 1,000 days have been highlighted as a window of opportunity to alter a child's cognitive results. ${ }^{4}$

The quality and stability of a child's human relationships in the early years lay the foundation for a wide range of later developmental outcomes, e.g. self-confidence, motivation to learn, achievement in school and later in life, the ability to control

\section{Corresponding author:}

Ni Ketut Susila Dharma Asih

Medical and Sciences Affair

Danone Specialized Nutrition Indonesia

Email:ni-ketut.asih@danone.com aggressive impulses and resolve conflicts in nonviolent ways, knowing the difference between right and wrong, capable to develop and sustain casual friendships and intimate relationships, and to be a successful parent oneself. ${ }^{1,5,6}$ Child development specialists across multiple disciplines acknowledge the importance of positive socialemotional development (SED) to overall child wellbeing. ${ }^{6}$ Therefore, this review provides an overview of key literature related to understanding and optimizing SED in early life. Then, we discuss the concept of gut-brain axis (GBA) and its role in critical window of early postnatal period, and its stimulations to increase the SED in children.

\section{Social-emotional development in early childhood}

Social-emotional development is defined as 'a child's developing capacity to (i) experience, manage and express the full range of positive and negative emotions; (ii) develop close, satisfying relationships with other children and adults; and (iii) actively explore their environment and learn. As 
social-emotional skills form the foundation for later functioning in school and for building lasting relationships with other people, the need to assess such skills in young children is now widely accepted. ${ }^{7}$

It is commonly assumed that infants have emotion concepts by about six months of age, as evidenced by their ability to discriminate between posed facial configurations that are stereotypes of facial expressions anger, sadness, fear, and happiness categories. ${ }^{8}$ A study examined infants' responding to others' emotional displays across 5 discrete emotions. The results revealed that infants use different functional behaviors in response to discrete emotions and were clearest in the 24-monthold infants. ${ }^{9}$

Psychopathology in infants and toddlers (ages 0-24 months old) is often found within the socialemotional domains and tends to persist over time. ${ }^{7}$ Psychopathology in early childhood is associated with concurrent global impairment, and there is evidence for specific relationships between disorders and functioning domains; young children with autism spectrum disorder (ASD) and oppositional defiant disorder show impairment in peer relationships, family functioning and parentchild relationships, and behavioral functioning. ${ }^{10}$

\section{Physiology of social-emotional development}

Social interaction is governed by several subcortical forebrain structures such as the prefrontal cortex (PFC), anterior cingulate cortex, amygdala (AMG), hippocampus, and hypothalamus, which form part of an integral interconnected network to facilitate this complex behavior. Damage or dysfunction to any one of these brain regions can give rise to perturbations in social behavior. Indeed, the neurobiology of regions such as the AMG and PFC have been shown to be altered in disorders of the social brain such as autism spectrum disorders (ASDs). ${ }^{11}$

The key brain region for social functioning is the AMG. Damage to the AMG impairs individuals' abilities to recognize complex social emotions in facial expressions. AMG volume and functional connectivity with cortical regions correlates with social network size in young adults, and alterations to AMG circuitry contribute to social processing deficits in many disorders, such as ASDs and anxiety disorders. ${ }^{12}$

Neuronal circuits in the brain are shaped by experience during 'critical periods' in early postnatal life. ${ }^{13} \mathrm{~A}$ young and developing brain has an increased flexibility of the circuitry. Once a particular circuitry pattern becomes established, it is difficult for the effects of new and different experiences to alter that architecture. This means that early experience has a unique advantage in shaping the architecture of developing brain circuits before they are fully mature and stabilized (Fig.1). ${ }^{14}$

\section{How to optimize social emotional development in early life}

It was recently discovered that children who are institutionalized at birth have IQs in the low 70s, despite the fact that they are otherwise developing normally. However, placing such children in high quality foster care before the age of two years

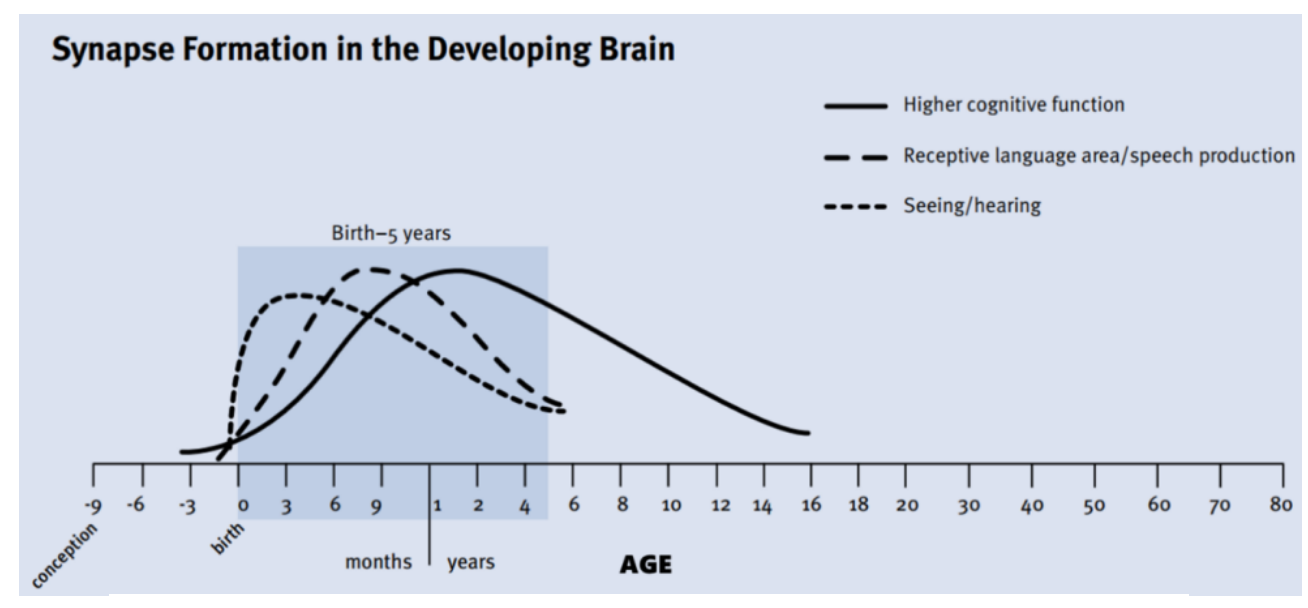

Figure 1. Synapse Formation in the Developing Brain 
increases IQ significantly. A similar trend also occurs for language, and the development of the electroencephalogram (EEG) power and coherence. The influence of childhood environment is far more evident in such basic cognitive processes as sensory perception, and the foundation of brain architecture must lay in the early formative years. ${ }^{15}$

It is important that experiences provided in the earliest years are appropriate for the young child's stage of development. Reading a picture book with a toddler who is learning to speak, for example, provides an important opportunity to point to and talk about the pictures, not to focus on the written words. The ability to decode written language comes later, when the appropriate, higher level brain circuitry will be built. Because low-level circuits mature early and high-level circuits mature later, different kinds of experiences are critical at different ages for optimal brain development, a concept called age-appropriate experience. Soon after birth, basic sensory, social, and emotional experiences are essential for optimizing the architecture of low-level circuits. At later ages, more sophisticated kinds of experiences are critical for shaping higher-level circuits. When adults or communities expect young children to master skills for which the necessary brain circuits have not yet been formed, they waste time and resources, and may even impair healthy brain development by inducing excessive stress in the child. ${ }^{14}$

\section{Gut-brain axis (GBA)}

In the last several years, interest in the interactions among the gut microbiome, brain, and behavior has exploded. ${ }^{11,16}$ The microbes residing in our gut harvest energy from the food we eat, train our immune system, and release metabolites and hormones important for regulating our physiology. Chemical signals from our microbiota can act locally within the gut, and can also have larger systemic effects. ${ }^{17}$

Preclinical evidence supports a role of the gut microbiome in behavioral responses associated with pain, emotion, social interactions, and food intake. Converging evidence suggests that the brain and the gut microbiota are in bidirectional communication. ${ }^{16}$ Collectively, this bidirectional pathway is known as the microbiota-GBA. ${ }^{11,18,19}$ Interactions between gut microbes and established psychoneuroimmunologic pathways allow for gut-brain communication., including immunological (cytokines), endocrine (hypothalamic-pituitary-adrenal), and neural (vagus) pathways. ${ }^{20-22}$

Both the gut microbiota and the central nervous system undergo fast changes throughout the first two years of life. ${ }^{20}$ The early window for gut microbial establishment is critical. Mode of delivery is known to play a significant role in the natural assembly of the neonate gut microbiota in term infants, where the pioneer colonizers of vaginally delivered full term infants resemble the mother's vaginal microbiota. ${ }^{23}$ Advances in sequencing technologies are revealing that the early developmental microbiota signature influences almost every aspect of the organism's physiology. ${ }^{21}$

To date, there is limited high-quality evidence regarding alterations in microbial ecology or production of microbial-derived metabolic products in human patients with brain or brain gut disorders. For example, there is inconclusive evidence from human studies regarding the beneficial effects of manipulating the microbiota with prebiotics and antibiotics in patients with irritable bowel syndrome, even though meta-analyses suggest a small therapeutic effect for probiotics. Furthermore, it is not clear whether alterations observed in the microbiota of patients with these disorders arise from primary alterations at the gut microbial interface (bottom-up effects) and/or changes in brain-gut signaling (top-down effects). ${ }^{16}$

\section{Clinical findings of gut-brain axis theory}

A number of experimental approaches have been employed to study the modulatory effects of gut microbiota on gut-brain interactions in experimental animals, including treatment with antibiotics, fecal microbial transplant, germ-free (GF) animal models, and treatment with probiotics. Microbiota-related effects have been reported in relation to anxiety-like behavior, depression-like behavior, nociceptive responses, stress responsiveness, feeding behavior, and taste preferences. Despite the extensive remodeling of biological systems in the GF animal, the fact that some observed behaviors and brain changes could be reversed by reconstitution of pathogen-free microbiota (conventionalization) 
validates some of the conclusions drawn. ${ }^{16,18}$ Nevertheless, as the GF animal has no counterpart in human brain development, premature conclusions about the translational relevance of these findings to humans should be avoided. ${ }^{16}$

Ongoing recognition of the role of the gut microbiota in preclinical models of disease, especially neuropsychiatric, demands evaluation in clinical settings. ${ }^{16}$ ADHD is the most prevalent neurodevelopmental disorder and is highly heritable with other contributing environmental factors such as diet and microbiome also influencing risk. ADHD individuals had an increase of Actinobacteria mainly at the expense of Firmicutes and Bifidobacterium within the phylum Actinobacteria compared to the healthy controls. ${ }^{23}$

There is a high heterogeneity in the findings of altered microbial profiles in ASD. It is reported that there are alterations in the microbial communities in ASD but conflicting results are reported in the prevalence of Firmicutes, Bacteroidetes and Proteobacteria, Bifidobacterium, Clostridium, and Bifidobacterium between children with ASD and controls. The incidence of schizophrenia is positively correlated with preterm birth and associated with microbiota changes, suggesting that schizophrenia is a neuropsychiatric disorder where microbiome early in life might be involved in the disease process. ${ }^{23}$

\section{Increasing the social-emotional development via} gut-brain axis

Throughout one's lifetime, the gut-brain, brain, and mentality develop practically in lockstep. (Fig.2). Improvement of psychology, neuroscience, and psychiatry will all benefit from gut-brain psychology. Various microbiota-improving methods including fecal microbiota transplantation (FMT), probiotics, prebiotics, a balance and healthy diet, also healthy lifestyle have all been demonstrated to improve the function of the gutbrain, microbiota-GBA, and brain. In the future, it will be feasible to utilize the gut microbiota to improve brain and mental health, as well as to prevent and treat disorders associated with them. ${ }^{19}$

Pathogen infection quickly induces sickness behavior, with infected subjects showing fatigue, social avoidance, decreased appetite, and increased anxiety-like behavior. Supplementing with certain probiotics, ${ }^{24}$ prebiotics, or fermented foods reduces negative behaviors and improves these emotions. ${ }^{19}$ Surgency and extraversion are linked to phylogenetic diversity in toddlers. ${ }^{25}$ A healthy microbiota helps the host cope with stress, whereas an aberrant microbiome lowers resistance and makes the host more susceptible to stress-related ailments. ${ }^{19}$

Gut-brain psychology will play a role to the development of general psychology, such as character, memory, and behavior. Its clinical application, such as managing the brain and behavior through gut microbiota intervention, is likely to have a greater impact.. There are mainly seven recognized microbiota interventions: the GF technique, pathogen infection, antibiotics, FMT, ${ }^{26}$ probiotics, ${ }^{24}$ prebiotics, and diet; All of the methods have showed considerable promise in terms of mind and behavior regulation.. Among these methods, the first two are only possible in animals' experiments, whereas the third one is commonly utilizedin antiinfection treatments, and the last four are all promising in microbiota improvement. ${ }^{19}$

The healthy diet promotes the function of the microbiota-GBA and leads to improvements in health and well-being. ${ }^{19}$ Nutritional psychology will connect the microbiota-GBA with psychology. ${ }^{27}$ Food is the most important component affecting the gut microbiota, and it has an impact that lasts a lifetime. Through the microbiota-GBA, a balance and healthy diet leads to a healthy gut microbiota and gut-brain, and supports brain and mental health. ${ }^{19}$

Sarris et al. (2015) suggested nutritional psychiatry; they believed that diet is a key modifiable intervention target for prevention of the initial incidence of common mental disorders. Convincing data suggest that select nutrient-based supplements (in isolation, or in combination, e.g. omega-3 fatty acids, zinc, B vitamins, and vitamin D), might provide neurochemical modulatory activities that are beneficial in the management of mental disorders (e.g. bipolar depression, posttraumatic stress disorder, and major depression). ${ }^{28}$ The happiness and quality of life among caregiver and families/parents are also studied plays role in improving the child's gut health and effectiveness of nutrition intervention, hence need to be put into 
serious consideration in the future. ${ }^{29}$ Moreover, combining diet therapy with other interventions, including drug treatment, psychotherapy, and exercise, has shown some good effects in mental therapy. ${ }^{19}$

\section{Conclusion}

During the first 1,000 days of life, children learn progressively to regulate their impulses, get along with others, understand and respond to emotions, focus their attention on salient stimuli, follow rules, and engage in culturally appropriate social interactions. Negative early experiences can impair children's mental health and effect their cognitive, behavioral, social-emotional development.

The GBA pathway suggests that microbial signals are important for healthy neurodevelopment and programming of social behaviors in the brain. It is noteworthy that the microbiota is more "medically" accessible and modifiable than the human genome. This fact provides a promising opportunity for preventing or treating neuropsychiatric conditions.

\section{Conflict of Interest}

Authors declared no conflict of interest regarding this article.

\section{Open Access}

This article is distributed under the terms of the Creative Commons Attribution 4.0 International Licence

(http://creativecommons.org/licenses/by/4.0/), which permits unrestricted use, distribution, and reproduction in any medium, provided you give appropriate credit to the original author(s) and the source, provide a link to the Creative Commons license, and indicate if changes were made.

\section{References}

1. McCoy DC, Cuartas J, Waldman M, Fink G. Contextual variation in young children's acquisition of socialemotional skills. PLoS ONE. 2019:14(11): e0223056

2. Reijneveld SA, Hielkema M, Stewart RE, de Winter AF. The added value of a family-centered approach to optimize infants' social-emotional development: A quasi-experimental study. PloS ONE. 2017;12(12):e0187750

3. Janice L. Cooper RM, Jessica Vick. Social-emotional Development in Early Childhood What Every Policymaker Should Know 2009. Available from: https://core.ac.uk/download/pdf/161435199.pdf.

4. Jena A, Montoya CA, Mullaney JA, Dilger RN, Young W, McNabb WC, et al. Gut-Brain Axis in the Early Postnatal Years of Life: A Developmental Perspective. Frontiers in integrative neuroscience. 2020;14:44.

5. Child NSCoTD. Young Children Develop in an Environment of Relationships 2004. Available from: https://developingchild.harvard.edu/wpcontent/uploads/2004/04/Young-Children-Develop-inan-Environment-of-Relationships.pdf.

6. Kristen E. Darling-Churchill LL. Early childhood social and emotional development' Advancing the field of measurement. Journal of Applied Developmental Psychology. 2016;45:7

7. Pontoppidan M, Niss NK, Pejtersen JH, Julian MM, Vaever MS. Parent report measures of infant and toddler social-emotional development: a systematic review. Family practice. 2017;34(2):127-37

8. Hoemann K, Xu F, Barrett LF. Emotion words, emotion concepts, and emotional development in children: A constructionist hypothesis. Developmental psychology. 2019;55(9):1830-49

9. Walle EA, Reschke PJ, Camras LA, Campos JJ. Infant differential behavioral responding to discrete emotions. Emotion. 2017;17(7):1078-91.

10. Finsaas MC, Kessel EM, Dougherty LR, Bufferd SJ, Danzig AP, Davila J, et al. Early Childhood Psychopathology Prospectively Predicts Social Functioning in Early Adolescence. Journal of clinical child and adolescent psychology : the official journal for the Society of Clinical Child and Adolescent Psychology, American Psychological Association, Division 53. 2020;49(3):353-64

11. Sherwin E, Bordenstein SR, Quinn JL, Dinan TG, Cryan JF. Microbiota and the social brain. Science. 2019;366(6465)

12. Johns CB, Lacadie C, Vohr B, Ment LR, Scheinost D. Amygdala functional connectivity is associated with social impairments in preterm born young adults. NeuroImage Clinical. 2019;21:101626

13. Hensch TK. Critical period plasticity in local cortical circuits. Nature reviews Neuroscience. 2005;6(11):87788.

14. Child NSCoTD. The Timing and Quality of Early Experiences Combine to Shape Brain Architecture 2008. Available from: https://developingchild.harvard.edu/wpcontent/uploads/2007/05/Timing_Quality_Early_Expe riences-1.pdf.

15. Fox SE, Levitt P, Nelson CA, 3rd. How the timing and quality of early experiences influence the development of brain architecture. Child development. 2010;81(1):28-40. 
16. Martin CR, Mayer EA. Gut-Brain Axis and Behavior. Nestle Nutrition Institute workshop series. 2017;88:4553.

17. Duvallet C, Gibbons SM, Gurry T, Irizarry RA, Alm EJ Meta-analysis of gut microbiome studies identifies disease-specific and shared responses. Nature communications. 2017;8(1):1784.

18. Luk B, Veeraragavan S, Engevik M, Balderas M, Major A, Runge J, et al. Postnatal colonization with human "infant-type" Bifidobacterium species alters behavior of adult gnotobiotic mice. PloS ONE. 2018;13(5):e0196510.

19. Liang $\mathrm{S}, \mathrm{Wu} \mathrm{X}$, Jin F. Gut-Brain Psychology: Rethinking Psychology From the Microbiota-Gut-Brain Axis. Frontiers in integrative neuroscience. 2018;12:33.

20. Brennan PA, Dunlop AL, Smith AK, Kramer M, Mulle J, Corwin EJ. Protocol for the Emory University African American maternal stress and infant gut microbiome cohort study. BMC pediatrics. 2019;19(1):246.

21. Foster JA, Rinaman L, Cryan JF. Stress \& the gut-brain axis: Regulation by the microbiome. Neurobiology of stress. 2017;7:124-36.

22. Ganci M, Suleyman E, Butt H, Ball M. The role of the brain-gut-microbiota axis in psychology: The importance of considering gut microbiota in the development, perpetuation, and treatment of psychological disorders. Brain and behavior. 2019;9(11):e01408.

23. Lu J, Claud EC. Connection between gut microbiome and brain development in preterm infants. Developmental psychobiology. 2019;61(5):739-51.

24. Cryan JF, Dinan TG. Mind-altering microorganisms: the impact of the gut microbiota on brain and behaviour. Nature reviews Neuroscience. 2012;13(10):701-12.

25. Christian LM, Galley JD, Hade EM, Schoppe-Sullivan S, Kamp Dush C, Bailey MT. Gut microbiome composition is associated with temperament during early childhood. Brain, behavior, and immunity. 2015;45:118-27.

26. Aroniadis OC, Brandt LJ. Fecal microbiota transplantation: past, present and future. Current opinion in gastroenterology. 2013;29(1):79-84.

27. Allen AP, Dinan TG, Clarke G, Cryan JF. A psychology of the human brain-gut-microbiome axis. Social and personality psychology compass. 2017;11(4):e12309.

28. Sarris J, Logan AC, Akbaraly TN, Amminger GP, Balanza-Martinez V, Freeman MP, et al. Nutritional medicine as mainstream in psychiatry. The lancet Psychiatry. 2015;2(3):271-4.

29. Bardosono S, Hildayani R, Chandra DN, Wibowo Y, Basrowi RW. Bonding Development Between Parents and Children Through Playing Together to Improve Happiness. Nutrition Journal World. 2017;.1( 1):41-51 